Article

\title{
Towards Performant Design of Carbon-Based Nanomotors for Hydrogen Separation through Molecular Dynamics Simulations
}

\author{
Sebastian Muraru ${ }^{1,2}$ and Mariana Ionita ${ }^{1,2, *}$ \\ 1 Faculty of Medical Engineering, University Politehnica of Bucharest, GhPolizu 1-7, 011061 Bucharest, Romania; \\ sebmuraru@gmail.com \\ 2 Advanced Polymer Materials Group, University Politehnica of Bucharest, GhPolizu 1-7, \\ 011061 Bucharest, Romania \\ * Correspondence: mariana.ionita@polimi.it
}

Received: 10 November 2020; Accepted: 14 December 2020; Published: 16 December 2020

\begin{abstract}
Clean energy technologies represent a hot topic for research communities worldwide. Hydrogen fuel, a prized alternative to fossil fuels, displays weaknesses such as the poisoning by impurities of the precious metal catalyst which controls the reaction involved in its production. Thus, separating $\mathrm{H}_{2}$ out of the other gases, meaning $\mathrm{CH}_{4}, \mathrm{CO}, \mathrm{CO}_{2}, \mathrm{~N}_{2}$, and $\mathrm{H}_{2} \mathrm{O}$ is essential. We present a rotating partially double-walled carbon nanotube membrane design for hydrogen separation and evaluate its performance using molecular dynamics simulations by imposing three discrete angular velocities. We provide a nano-perspective of the gas behaviors inside the membrane and extract key insights from the filtration process, pore placement, flux, and permeance of the membrane. We display a very high selectivity case $\left(\omega=180^{\circ} \mathrm{ps}^{-1}\right)$ and show that the outcome of Molecular Dynamics (MD) simulations can be both intuitive and counter-intuitive when increasing the $\omega$ parameter $\left(\omega=270^{\circ} \mathrm{ps}^{-1} ; \omega=360^{\circ} \mathrm{ps}^{-1}\right)$. Thus, in the highly selective, $\omega=180^{\circ} \mathrm{ps}^{-1}$, only $\mathrm{H}_{2}$ molecules and 1-2 $\mathrm{H}_{2} \mathrm{O}$ molecules pass into the filtrate area. In the $\omega=270^{\circ} \mathrm{ps}^{-1}, \mathrm{H}_{2}, \mathrm{CO}, \mathrm{CH}_{4}$, $\mathrm{N}_{2}$, and $\mathrm{H}_{2} \mathrm{O}$ molecules were observed to pass, while, perhaps counter-intuitively, in the third case, with the highest imposed angular velocity of $360^{\circ} \mathrm{ps}^{-1}$ only $\mathrm{CH}_{4}$ and $\mathrm{H}_{2}$ molecules were able to pass through the pores leading to the filtrate area.
\end{abstract}

Keywords: hydrogen separation; rotating carbon nanotube membrane; molecular dynamics

\section{Introduction}

Predominantly, hydrogen meant to be used as fuel is obtained through steam reforming gas, with the following chemical reactions involved $\left[\mathrm{CH}_{4}+\mathrm{H}_{2} \mathrm{O} \rightarrow \mathrm{CO}+3 \mathrm{H}_{2} ; \mathrm{CO}+\mathrm{H}_{2} \mathrm{O} \rightarrow \mathrm{CO}_{2}+\mathrm{H}_{2}\right]$. Any impurities, such as $\mathrm{CH}_{4}, \mathrm{CO}_{2}, \mathrm{CO}, \mathrm{N}_{2}$, and $\mathrm{H}_{2} \mathrm{O}$ molecules can significantly reduce the performance of a hydrogen fuel cell by poisoning its precious metal catalyst driving forward the electrochemical reactions $[1,2]$. Thus, separating the $\mathrm{H}_{2}$ molecules out of the mixture of gases is crucial for keeping fuel cells efficient. Mature separation technologies such as pressure swing absorption and cryogenic distillation have lost ground to newer solutions, e.g., separation membranes due to their affordability and easy operation [2-5]. Many of these have been designed using different metals, silica, zeolite, various polymers, and carbon, yet without coming upon an ideal solution [1,3-7].

Carbon, as one of the most common and versatile elements in the universe, has continuously attracted significant interest within the scientific community over the span of several decades. Constant discovery of new allotropes, each displaying unique properties, allowed for carbon to be used for an extraordinary array of purposes and applications $[8,9]$. In particular, synthetic allotropes 
such as the one-dimensional carbon nanotubes [10] and the two-dimensional graphene [11] have ignited enormous interest within the materials science community. Despite being impermeable to water and gas molecules, techniques such as ion bombardment or e-beam lithography $[3,12]$ can be used to create tailored nano-pores and permeabilize these structures.

Molecular dynamics (MD) simulations represent a computational technique useful in acquiring a nano, atom-level perspective of all molecules making up a defined system. Complete trajectories are built step-by-step for each atom present in a simulation box. Although it is used ultimately to complement experimental research, it may also be used to uncover key insights entirely on its own. This is possible because a computational researcher can explore scenarios that may prove too costly, time-consuming, or simply inaccessible yet to experimental research. Here, we make use of MD simulations to explore certain useful parameters during hydrogen separation using a theoretical framework. Several MD-only studies have investigated the molecular mechanisms useful in describing the separation potential of carbon-based structures, either for gas separation [12-18] or water desalination $[19,20]$.

The recently observed rotational motion of double-walled carbon nanotubes exposed to an electric field [21] opened the door to more complex MD simulations of carbon-based nanomotors, investigating areas such as behavior in water, water transport, and desalination [21-24]. To our knowledge, there are no existing MD studies on carbon-based nanomotors evaluating gas separation. In this study, we explored the impact of an imposed angular velocity on the performance of $\mathrm{H}_{2}$ separation of a rotating partially double-walled carbon nanotube design out of a mixture of gases containing $\mathrm{H}_{2}, \mathrm{CH}_{4}, \mathrm{CO}, \mathrm{CO}_{2}, \mathrm{~N}_{2}$, and $\mathrm{H}_{2} \mathrm{O}$ molecules. The gases, placed inside the rotating carbon nanotube could either exit through the nanopores present along the length of the nanotube or through one of its ends which was left open. Performance was determined by analyzing the filtrated molecules, pore placement, and exploring parameters such as flux and permeance. In a practical setting, the angular velocity imposed on the rotating carbon nanotube can be generated by the presence of an electric field, either directly or through a gear-based mechanism, for which we have provided a segment on our design, leaving it pore-free. We found a favorable case of imposed angular velocity leading to impressive selectivity $\left(\omega=180^{\circ} \mathrm{ps}^{-1}\right)$ and showed that the outcome of MD simulations can be both intuitive and counter-intuitive when increasing the $\omega$ parameter $\left(\omega=270^{\circ} \mathrm{ps}^{-1} ; \omega=360^{\circ} \mathrm{ps}^{-1}\right)$. The design allows for the gas mixture to be exposed to a high surface area; each gas molecule always traveling towards a pore. $\mathrm{H}_{2}$ molecules were able to quickly exit the rotating nanotube by the 10ns mark and no gas molecules were able to cross back into the membrane leading to overall increased efficiency. In addition, flux values were comparable to graphene pores corresponding to 10-16 removed atoms. Overall, we have shown that the angular velocity plays a significant role in hydrogen separation through a rotating nanotube and discussed key parameters involved in the separation process.

\section{Results and Discussion}

\subsection{Choosing Pore Shape and Size}

In this study, the pores' shapes and sizes, shown in Figure 1A,B, were chosen based on our previous investigation on gas separation membranes [18]. The larger pore, corresponding to the removal of 14-atoms out of a graphene sheet, was proven to allow all molecules of the described gas mixture to pass through, while the smaller pore, corresponding to the removal of a 6-atom graphene ring, was shown to allow only the passage of $\mathrm{H}_{2}$ and $\mathrm{H}_{2} \mathrm{O}$ molecules.

An important aspect to mention when conducting investigations similar to ours, obvious when looking at Figure $2 \mathrm{~A}-\mathrm{C}, \mathrm{G}-\mathrm{I}, \mathrm{M}-\mathrm{O}$, is that some deformation of the carbon nanotube occurred during each of the simulations due to the imposed rotational movements. This aspect can be observed easiest by looking at the no gas area. The same area is specifically shown in Figure 3. These structural deformations can lead to changes that occur in the shape and size of the pores situated on the rotating carbon nanotube and thus different gas separation behaviors. In addition, looking at Figure 2D-F,J-L,P-R, no gas molecules 
ever cross back into the membrane. The small fluctuations seen in the case of $\mathrm{H}_{2}$ molecules are due to the calculation method (the $\mathrm{H}_{2}$ number on the graphs always goes back up quickly).

A

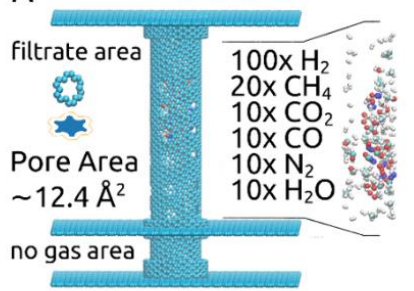

B

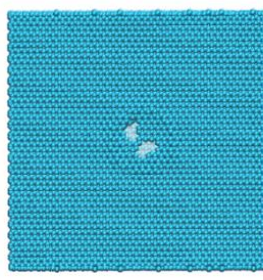

C

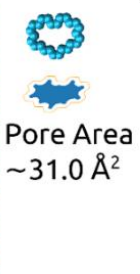

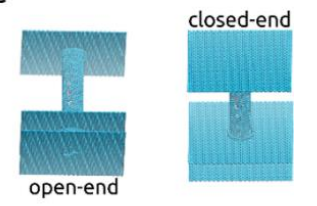

Figure 1. (A) Outline of the rotating carbon nanotube membrane, together with gas mixture and small pore details; (B) View of the open-end together with the larger pore details; (C) Perspectives on the membrane showing the open-end and the closed-end.

A

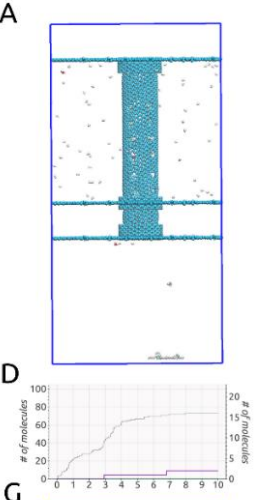

G

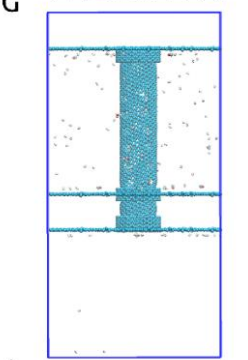

$J$

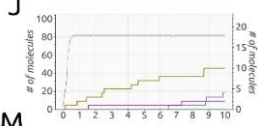

M

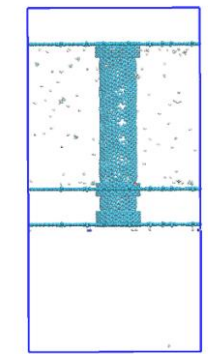

$P$

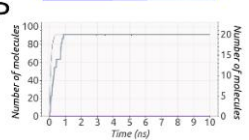

B

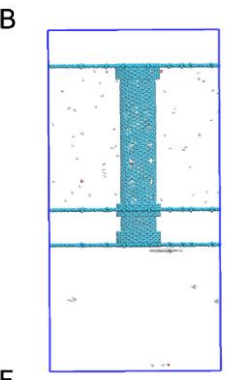

E

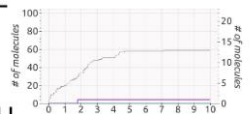

$\mathrm{H}$

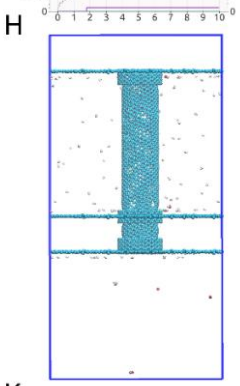

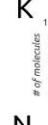

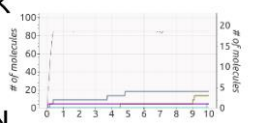

$\mathrm{N}$

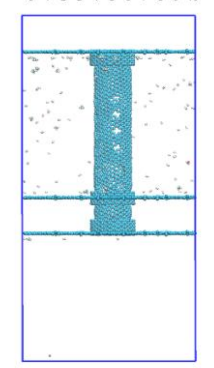

Q

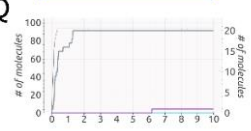

C

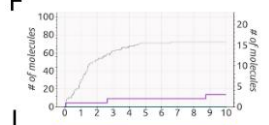

I
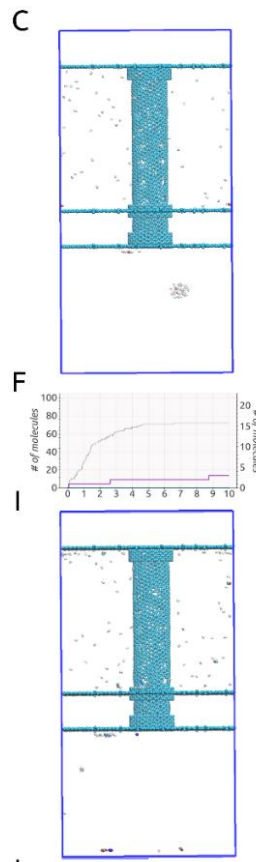

$\mathrm{L}$

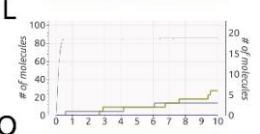

$\mathrm{O}$

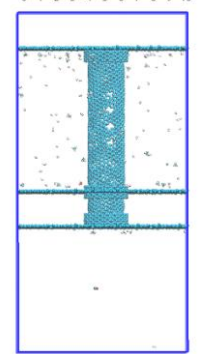

R

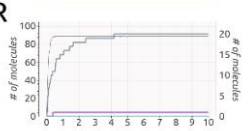

Figure 2. (A-C). $\left(\omega=180^{\circ} \mathrm{ps}^{-1}\right),(\mathbf{G}-\mathbf{I}) \cdot\left(\omega=270^{\circ} \mathrm{ps}^{-1}\right),(\mathbf{M}-\mathbf{O}) \cdot\left(\omega=360^{\circ} \mathrm{ps}^{-1}\right)$ Final frame of the MD simulations (10 ns); (D-F). $\left(\omega=180^{\circ} \mathrm{ps}^{-1}\right),(\mathbf{J}-\mathbf{L}) .\left(\omega=270^{\circ} \mathrm{ps}^{-1}\right),(\mathbf{P}-\mathbf{R}) .\left(\omega=360^{\circ} \mathrm{ps}^{-1}\right)$ Evolution of the number of molecules passing into the filtrate area during simulations time. 

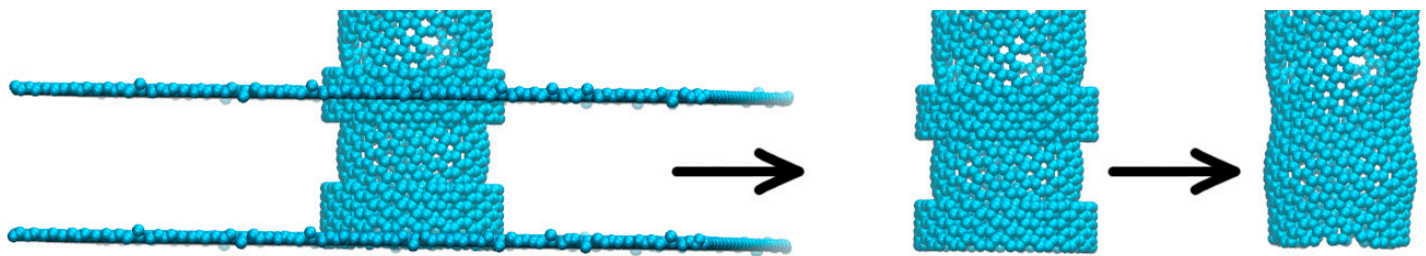

Figure 3. Example of the structural deformation occurring in the rotating carbon nanotube due to imposed angular velocity.

Effective pore areas $A_{p}$ were determined using a Monte Carlo hit-and-miss procedure $[14,16,18]$, considering the effective carbon atom radius $R_{\text {eff }}=R_{m, c} / \sqrt{ } 2$ and $R_{m, c}$ equal to $0.17 \mathrm{~nm}$. The approximate results are shown numerically in Figure $1 \mathrm{~A}, \mathrm{~B}$, meaning $\sim 12.4 \AA^{2}$ for the smaller pores located along the carbon nanotube and $\sim 31.0 \AA^{2}$ for the larger pores placed in the graphene layer at one of its ends. Blue-filled shapes for each pore type showing their effective pore areas are also shown in Figure 1A,B (images not to scale). The two values were calculated considering the pores to be sculpted in a planar 2D graphene sheet. This approach fits very well in the case of the larger pore. For the smaller pore, however, determining the area in such a manner is less accurate due to being sculpted in a carbon nanotube with a chiral vector determined by the indices $(12,16)$. Nevertheless, it still provides a useful approximate value. Using the same method for calculating the smaller pore's area, now when sculpted in the chiral nanotube, we ended up with the following approximate values: $12.26 \AA^{2}$ at $0 \mathrm{~ns}$ before the start of the simulations, $12.51 \AA^{2}$ for $\omega=180^{\circ} \mathrm{ps}^{-1}, 13.02 \AA^{2}$ for $\omega=270^{\circ} \mathrm{ps}^{-1}$ and $14.70 \AA^{2}$ for $\omega=360^{\circ} \mathrm{ps}^{-1}$.

Thus, given the imposed angular velocities to the carbon nanotube, changes in the shape and size of the smaller pores took place. The shape and size of the pores, together with the angular velocity of the nanotube are the main parameters behind the distinct results obtained here for each $\omega$ case. This aspect helps explain the results obtained in this paper. The influence of an imposed angular velocity on the geometry of a carbon nanotube and pores sculpted on its surface can be investigated in future studies.

The rationale behind keeping an "open" end to the membrane, represented by the graphene layer with the large pores placed as pictured in Figure 1A,B, was to allow for the gas mixture to be fed into the carbon nanotube. This obviously led to some $\mathrm{H}_{2}$ molecules to be lost as they were able to exit the membrane through the pores situated at the end of the tube instead of crossing through the smaller pores into the filtrate area. However, we deemed this "open" end setup more realistic and practical. Given our previous results [18], which confirmed that all gas molecules present in the gas mixture used here are able to pass through the $31.0 \AA^{2}$ pore, we focused the current investigation on the separation process that would occur once the gas mixture is already loaded into the rotating carbon nanotube membrane and the effect of the imposed angular velocity.

\subsection{Simulation Results}

Curiously, given the setups described, all three $\omega$ cases yielded significantly different results, consistent throughout all three runs, as can be observed in Figure 2. We must mention that in all nine simulations, all $\mathrm{H}_{2}$ molecules had either crossed through the smaller pores or the larger pores by the $10 \mathrm{~ns}$ mark. Thus, all of them had left the rotating carbon nanotube by the time-point at which we stopped our simulations. On top of that, given the three tested values for the imposed angular velocity, at least $60 \%$ of the $\mathrm{H}_{2}$ molecules were able to cross into the filtrate area within the simulated time in all given setups.

\subsection{Angular Velocity $\omega=180^{\circ} \mathrm{ps}^{-1}$}

In the case of $\omega=180^{\circ} \mathrm{ps}^{-1}$, consistent with our previous investigations [18], only $\mathrm{H}_{2}$ and $\mathrm{H}_{2} \mathrm{O}$ molecules were able to pass through the smaller pores. The process of $\mathrm{H}_{2}$ molecules passing through the smaller pores and entering the filtrate area took place in about $7-8 \mathrm{~ns}$, with $\sim 20 \mathrm{H}_{2}$ molecules passing 
within the first nanosecond and some other 40 to 50 molecules within the next 6-7 ns. In regards to $\mathrm{H}_{2} \mathrm{O}$ molecules, only 1-2 molecules were able to pass into the filtrate area, while most of them (6-7) were still found inside the carbon nanotube at the end of the $10 \mathrm{~ns}$. The reason for this is that although the smaller pores should theoretically allow them to pass through, these are found to cluster together inside the nanotube due to hydrogen bonding. In all our simulations this prevents most of them from leaving the rotating membrane, given the pore sizes used. No other gas molecules were able to pass through the smaller pores at this angular velocity.

Taking a look at the process of separation occurring throughout our simulations, we observed that some of the gas molecules inside the nanotube position themselves as if blocking the smaller pores and remain positioned so, despite the rotating motion. This aspect is shown in Supplementary Video S1 and in Figure $4 \mathrm{~A}$ with $\mathrm{CH}_{4}, \mathrm{CO}, \mathrm{H}_{2} \mathrm{O}, \mathrm{N}_{2}$, and $\mathrm{H}_{2}$ molecules blocking some of the pores. We also show, in Figure 4B, that by the end of the simulation, at 10ns, pores were still blocked by the larger molecules at a time at which $\mathrm{H}_{2}$ molecules were no longer found inside the rotating nanotube. We think that due to the imposed rotational motion and the size and shape of the chosen smaller pores, the smaller molecules such as $\mathrm{H}_{2}$ and $\mathrm{H}_{2} \mathrm{O}$ are able to eventually exit through the pores they are blocking. Thus, with an imposed $\omega$ of $180^{\circ} \mathrm{ps}^{-1}$, some degree of fouling will occur, leaving fewer pores open for $\mathrm{H}_{2}$ molecules to cross and leading more to pass through the open end of the membrane in exchange for the high selectivity offered. Between 59 and 73 out of $100 \mathrm{H}_{2}$ molecules were able to pass into the filtrate area throughout the three simulations with an imposed angular velocity of $180^{\circ} \mathrm{ps}^{-1}$. Interestingly, no $\mathrm{CO}_{2}$ molecules were found to block any of the smaller pores throughout all simulations but were instead able to pass through the larger pores as can be seen in Figure 2I.
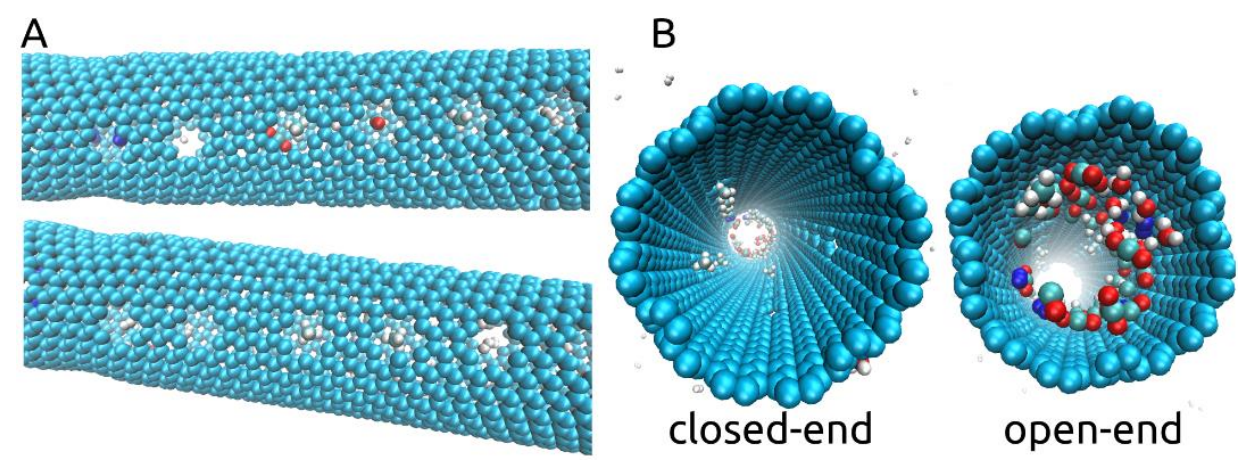

Figure 4. (A). Cases of $\mathrm{N}_{2}, \mathrm{CH}_{4}, \mathrm{CO}$, and $\mathrm{H}_{2}$ gas molecules shown to block some of the pores situated along the rotating central carbon nanotube; $(\mathbf{B})$. Closed-end and open-end views at the end of the simulations $\left(\omega=180^{\circ} \mathrm{ps}^{-1}\right)$ showing the agglomeration of the molecules at one of the ends and the $\mathrm{CH}_{4}$ molecules still blocking some of the pores.

At $\omega=180^{\circ} \mathrm{ps}^{-1}$, as it can be observed in Supplementary Video S1, some $\mathrm{H}_{2}$ adsorption to the wall of the rotating carbon nanotube took place, which allowed the gas molecules to travel with the nanotube in its rotating motion. However, the phenomenon lasted for a short time (a few ps) before the adsorbed molecules were disturbed. We think both the imposed high angular velocity and the placement of the pores along the membrane, with each pore crossing disturbing the nearby gas molecules, help prevent adsorption from taking place and thus allow all $\mathrm{H}_{2}$ molecules to leave the nanotube by the end of the simulation.

Another important aspect to discuss in the presented rotating carbon nanotube membrane design regards the placement of the pores leading to the filtrate area along the tube. In our case, as shown in Figure 1, we have placed the 24 smaller pores in the central part of the nanotube, leaving both ends with no pores to the filtrate area. As shown in Figure 5, at the end of all simulations (10 ns), unless found blocking one of the smaller pores, all molecules that remained in the rotating nanotube can be seen gathered at one of its ends. Without smaller pores nearby, the agglomeration of molecules becomes somewhat "stagnant" despite the rotational movement of the tube (see Supplementary Video S2). 
This aspect may be beneficial to the flux of $\mathrm{H}_{2}$ to the filtrate area as the smaller pores are then less likely to be blocked.
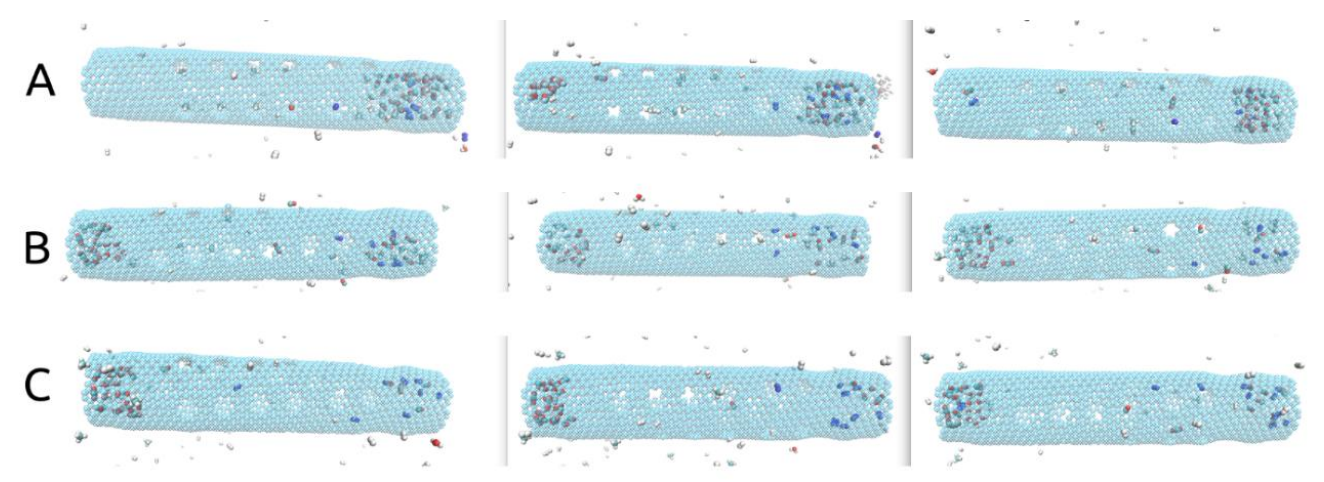

Figure 5. (A) $\left(\omega=180^{\circ} \mathrm{ps}^{-1}\right),(\mathbf{B})\left(\omega=270^{\circ} \mathrm{ps}^{-1}\right),(\mathbf{C})\left(\omega=360^{\circ} \mathrm{ps}^{-1}\right)$ Representations of the rotating central carbon nanotube at $10 \mathrm{~ns}$ simulation time showing the gas molecules gathered at one of its ends.

Additionally, inspecting Figure 5, all $\mathrm{H}_{2}$ molecules exited by the 10ns mark, and only $\mathrm{CH}_{4}, \mathrm{CO}$, $\mathrm{CO}_{2}, \mathrm{~N}_{2}$, and $\mathrm{H}_{2} \mathrm{O}$ molecules (clustered) can be seen inside. To provide an explanation for this we looked at the movement of $\mathrm{H}_{2}$ molecules.

As shown in Supplementary Video S2, the molecules in the mixture with a smaller mass, meaning $\mathrm{H}_{2}, \mathrm{CH}_{4}$, and $\mathrm{H}_{2} \mathrm{O}$ were able to move slightly quicker when inside the rotating nanotube. On top of that, due to their small volume, which allowed them to "squeeze" through the larger molecules and their tendency to adsorb to the wall of the nanotube, $\mathrm{H}_{2}$ molecules had a high number of collisions. Together with the chirality of the nanotube, we think these aspects lead to the exiting of all $\mathrm{H}_{2}$ molecules within the $10 \mathrm{~ns}$ timeframe as they are more likely to travel along the nanotube and exit either towards the filtrate area or through the "open" end. The movement of $\mathrm{H}_{2}$ molecules within rotating carbon nanotubes of different chiralities could be investigated in future studies to determine whether chirality can drive the gas molecules due to their tendency to adsorb to the wall of the nanotube.

\subsection{Angular Velocity $\omega=270^{\circ} \mathrm{ps}^{-1}$}

For the higher imposed angular velocities, significantly different outcomes were observed compared to the $\omega=180^{\circ} \mathrm{ps}^{-1}$ case. The outcomes were consistent throughout all three repetitions corresponding to a certain $\omega$ value. In the case of $\omega=270^{\circ} \mathrm{ps}^{-1}, \mathrm{H}_{2}, \mathrm{CO}, \mathrm{CH}_{4}, \mathrm{~N}_{2}$, and $\mathrm{H}_{2} \mathrm{O}$ molecules were observed to pass through the smaller pores, as shown in Figure 2G-L. Almost all $\mathrm{H}_{2}$ molecules that passed into the filtrate area $(\sim 80-85)$ did so in the first half nanosecond. Again, no $\mathrm{H}_{2}$ molecules were found inside the tube at the end of the $10 \mathrm{~ns}$. Most molecules that passed through the smaller pores did so after blocking the pore for a while and exited either due to a collision with another gas molecule or due to the rotational motion of the nanotube, which allowed for slight changes in the orientation of the molecules. At this imposed angular velocity, despite that more $\mathrm{H}_{2}$ molecules reached the filtrate area, there was far less selectivity leading to a poor separation performance.

The movement of the $\mathrm{H}_{2}$ molecules inside the tube was slightly different compared to the $\omega=180^{\circ} \mathrm{ps}^{-1}$ case, please see Supplementary Video S3. Due to the higher imposed angular velocity, $\mathrm{H}_{2}$ molecules that were not blocking a pore were far less able to adsorb to the wall of the tube and travel in a synchronized manner with its rotational motion (as observed when $\omega=180^{\circ} \mathrm{ps}^{-1}$ ). Thus, due to the collisions with the wall, some $\mathrm{H}_{2}$ molecules traveling in a rotational motion nearby, did so both in the sense of the rotation and in the anti-sense, but slower when compared to the rotating wall. Essentially, this should have led to a higher number of collisions taking place inside the tube, which, alongside with the enlargement of the pores due to the larger angular velocity, enabled most molecules to exit into the filtrate area. $\mathrm{CH}_{4}$ molecules were also observed to pass through the pores after blocking it for a while and 
following a collision. No $\mathrm{CO}_{2}$ molecules were able to pass through the smaller pores. The results obtained for $\omega=270^{\circ} \mathrm{ps}^{-1}$ case are somewhat intuitive in that the higher angular velocity led to the expansion of the smaller pores and plummeted its selectivity while allowing more $\mathrm{H}_{2}$ molecules to pass into the filtrate area. From these simulations one can also see the following insight: as most remaining gas molecules at the 10ns mark are either found blocking one of the smaller pores or situated at one of the ends of the tube, the placement of the smaller pores along the center of the rotating carbon nanotube membrane could limit the passage of larger gas molecules into the filtrate area, thus improving the performance of the membrane.

\subsection{Angular Velocity $\omega=360^{\circ} \mathrm{ps}^{-1}$}

In the final case of $\omega=360^{\circ} \mathrm{ps}^{-1}$, as shown in Figure 2M-R, 89 to $94 \mathrm{H}_{2}$ molecules were able to pass through the smaller pores in less than $0.5 \mathrm{~ns}$. The $\mathrm{H}_{2}$ molecules that passed through the larger pores did so within $0.3 \mathrm{~ns}$ since the start of the simulations. Curiously, the only other gases which passed into the filtrate area at the end of the simulated time were $\mathrm{CH}_{4}$ and $\mathrm{H}_{2} \mathrm{O}$ molecules. Consistent throughout all three simulations with $\omega=360^{\circ} \mathrm{ps}^{-1}$, all $20 \mathrm{CH}_{4}$ molecules passed through the smaller pores and, similarly to the previously mentioned cases, $\omega=180^{\circ} \mathrm{ps}^{-1}$ and $\omega=270^{\circ} \mathrm{ps}^{-1}$, only one $\mathrm{H}_{2} \mathrm{O}$ molecule managed to do so, due to the clustering of the water molecules inside the tube. The fact that these results were consistent throughout all three repetitions shows, counter-intuitively, that using higher angular velocities values can lead to unique and unexpected insights. The fact that almost $90-95 \%$ of $\mathrm{H}_{2}$ molecules placed initially in the tube, together with $100 \%$ of the $\mathrm{CH}_{4}$ molecules and only $10 \%$ of the water molecules were able to pass into the filtrate area could be useful in future studies working on improving the current design by making use of a gradual separative process. In such a case, the second step would involve only the separation of $\mathrm{H}_{2}$ and $\mathrm{CH}_{4}$ molecules, as, curiously, no other gas molecules were able to pass into the filtrate area.

Observing the movement of the molecules inside the rotating carbon nanotube at $\omega=360^{\circ} \mathrm{ps}^{-1}$ shows the molecules with a smaller mass, $\mathrm{H}_{2}, \mathrm{CH}_{4}$, and $\mathrm{H}_{2} \mathrm{O}$ moving significantly faster than the larger $\mathrm{CO}, \mathrm{N}_{2}$, and $\mathrm{CO}_{2}$ molecules (see Supplementary Video $\mathrm{S} 4$ ). On top of that, due to collisions with the walls of the tube, the same smaller mass molecules rotate around their own center of mass much quicker than the larger ones, with $\mathrm{CH}_{4}$ being a special case and rotating faster than all linear molecules due to its geometry. Further on, given the quick rotation of the tube, no gas molecules were seen blocking any of the smaller pores or being able to adsorb to the rotating walls of the membrane. Thus, we think that when a gas molecule reaches the vicinity of a pore, due to the high angular velocity of the nanotube, the right orientation has to be found quickly in order to exit, which did not happen in the case of the larger linear molecules with a higher mass, but did take place in the case of the non-linear molecules with a smaller mass, meaning $\mathrm{CH}_{4}$ and $\mathrm{H}_{2} \mathrm{O}$ molecules. That is, gas molecules had a very short time to match an orientation that would have allowed them to cross the pore. After nearing a pore and colliding with the rotating wall, a small mass non-linear molecule was more likely to quickly rotate around its mass center and change its orientation quickly, which increased its chances of crossing. However, despite its linear structure, $\mathrm{H}_{2}$ molecules crossed the smaller pores easily due to their very small mass and volume as these aspects allowed them to change their orientation quickly and match that necessary to exit through the pore. More details, solely in regards to pore crossing can be reached in further investigations through ab initio molecular dynamics (AIMD) techniques.

\subsection{Flux and Permeance}

To further characterize the rotating carbon nanotube design for gas separation, we calculated the flux of $\mathrm{H}_{2}$ molecules through the smaller pores. Thus, in order to determine the total flux we counted the number of crossings from both inside and outside the tube and then made use of the formula below:

$$
\text { Flux }=\frac{\text { Crossing } s_{H 2} / N_{A}}{A_{\text {innersurface }} \times \text { Time }}
$$


where $N_{A}$ represents Avogadro's number, $A_{\text {inner_surface }}$ represents the inner surface of the tube and time corresponds to the time point of the last $\mathrm{H}_{2}$ crossing during the simulation. In addition, given that the majority of $\mathrm{H}_{2}$ molecules exit in a time window significantly shorter than the duration of the whole simulation, we have calculated the flux within the time frame in which $80 \%$ of $\mathrm{H}_{2}$ molecules had left the rotating carbon nanotube membrane. The inner surface of the tube was determined to be $59.854 \mathrm{~nm}^{2}$ using the formula $2 \pi \mathrm{rh}$. The radius of the tube, $\mathrm{r}$, was determined using the formula:

$$
\text { Radius }=\frac{a}{2 \pi} \times \sqrt{ }(m \times m+m \times n+n \times n)
$$

where $\mathrm{a}=0.246 \mathrm{~nm}$ and $\mathrm{m}$ and $\mathrm{n}$ represent the chiral indices of the nanotube. The results are shown in Figure 6A. Given the calculated total flux values, permeance values for our membrane design were estimated and shown both in $\mathrm{mol} / \mathrm{m}^{2} \mathrm{sPa}$ and gas permeation units (GPUs) in Figure 6B.
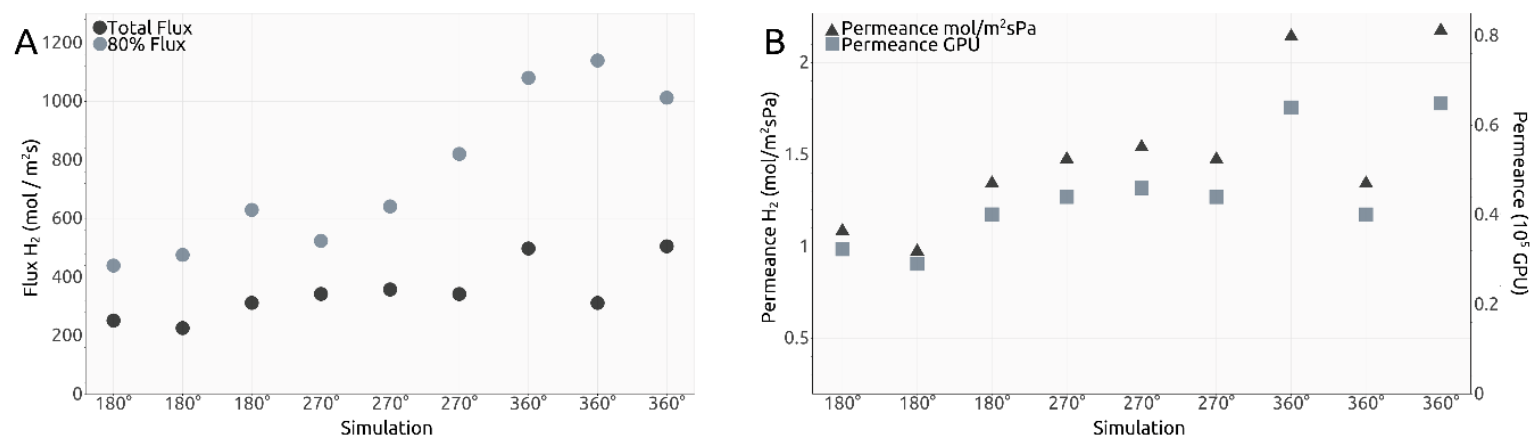

Figure 6. (A). Calculated total flux and $80 \%$ flux values; (B). Calculated permeance values in mol m${ }^{-2} \mathrm{~s}^{-1} \mathrm{~Pa}^{-1}$ and Gas Permeation Units (GPU).

Dividing the flux values presented in Figure 6A to the number of smaller pores present along the inner surface of the carbon nanotube, we obtain the intervals shown in Table 1:

Table 1. Resulting flux intervals after dividing flux values to the number of smaller pores.

\begin{tabular}{|c|c|c|}
\hline $\begin{array}{c}\text { Angular Velocity- } \omega \\
\text { (deg/ps) }\end{array}$ & $\begin{array}{l}\text { Total Flux } \\
\left(\mathrm{mol} / \mathrm{m}^{2} \mathrm{~s}\right)\end{array}$ & $\begin{array}{l}80 \% \text { Flux } \\
\left(\mathrm{mol} / \mathrm{m}^{2} \mathrm{~s}\right)\end{array}$ \\
\hline 180 & $9.0-13.0$ & $18.0-26.0$ \\
\hline 270 & $14.0-15.0$ & $21.5-34.0$ \\
\hline 360 & $13.0-21.0$ & $42.0-47.5$ \\
\hline
\end{tabular}

Despite the very different setups, comparing the total flux values obtained here (shown in Table 1) after division to the number of relevant pores, with the values presented in our previous research for a different design with the same gas separation purpose, the results are not significantly different from the interval $4.5-14.0 \mathrm{~mol} / \mathrm{m}^{2} \mathrm{~s}$ observed in [18] on the third filtration layer. The $80 \%$ flux intervals presented in Table 1 are higher, yet that is unsurprising, but these importantly highlight the manner in which the $\mathrm{H}_{2}$ molecules cross the smaller pores in the current design, with most of them managing to exit the rotating nanotube in the first $5.0 \mathrm{~ns}$ or $0.5 \mathrm{~ns}$ depending on the $\omega$ case, yet much quicker than the last $20 \%$.

Previous results were exceptional in terms of selectivity and permeability due to the gradual separation through multiple graphene layers each with its own specific pore size [18]. In the current design, we observed different behaviors, yet the exit of all $\mathrm{H}_{2}$ molecules took place in a very short time and no $\mathrm{H}_{2}$ molecules were left inside the nanotube at the end of the simulated $10 \mathrm{~ns}$. Thus, nanopores used for filtration can be customized for certain molecules not only by selecting their size and 2D shape but also by using an imposed angular velocity and the forces involved in the consequent rotational motion, thus changing its geometry. Therefore, using a rotating carbon nanotube membrane allowed 
the gas molecules to be exposed to most of the surface area of the membrane, uncovering interesting distinct behaviors, dependent on the imposed angular velocity. Nevertheless, alongside the advantage of the quick exiting of all $\mathrm{H}_{2}$ molecules within the membrane, a disadvantage of the current design is the loss of some of them due to the "open" end. Future studies may investigate different scenarios for improving the efficiency of the membrane. The easiest scenario to imagine being blocking the open end with a full graphene layer with no pores.

Comparing to Sun's results [16], the total flux values obtained in Figure 6A are comparable to a graphene pore size of 10 to 12 atoms, while the $80 \%$ flux values are comparable to a 12 -atom graphene pore for $\omega=180^{\circ} \mathrm{ps}^{-1}$, 14-atom graphene pore for $270^{\circ} \mathrm{ps}^{-1}$ and 16-atom graphene pore for $360^{\circ} \mathrm{ps}^{-1}$.

Naively comparing the flux values obtained for each $\omega$ case to each other, a clear trend can be seen showing that a higher imposed angular velocity leads to generally higher flux. However, an outlier can be seen in Figure $6 \mathrm{~A}$, with one total flux value in the $\omega=360^{\circ} \mathrm{ps}^{-1}$ significantly smaller than the other two. This was also one of the reasons we decided to calculate the $80 \%$ flux values. The corresponding value to the outlier for the $80 \%$ flux is higher than the two other similar simulations, which shows that most of the $\mathrm{H}_{2}$ molecules in the outlier simulation crossed into the filtrate area quicker and left the other $20 \%$ needing more time to cross due to, potentially, fewer collisions between molecules. Thus, with this explanation, we deem the outlier valid. One must take into account, however, that the heights of the total flux and the $80 \%$ flux values are not perfectly proportional due to events such as pore blocking, exit of other gas molecules, etc.

Similar to flux calculations, we estimated permeance values for the rotating carbon nanotube membrane, as shown in Figure 6B. Similar conclusions can be taken out of the permeance values as with the total flux calculations. We find all our simulations to indicate a value within the following intervals: 0.0097 to $0.0215 \mathrm{~mol}^{-3} / \mathrm{m}^{2} \mathrm{sPa}$ and 0.30 to $0.65 \times 10^{5} \mathrm{GPU}$, and thus in the vicinity of those calculated for our previous design, with values situated between 0.005 to $0.03 \mathrm{~mol}^{-3} / \mathrm{m}^{2} \mathrm{sPa}$ and 0.20 to $0.85 \times 10^{5} \mathrm{GPU}$, results already highlighted as superior to state-of-the-art solutions [18].

\section{Materials and Methods}

\subsection{Membrane Prototype}

The structure of the membrane is shown in Figure 1A,B: a central carbon nanotube with indices $(12,16)$ and $10 \mathrm{~nm}$ in length is held in place by two $10 \mathrm{~nm} \times 10 \mathrm{~nm}$ graphene sheets placed at its ends. At one end the graphene sheet is intact ("closed-end"), while at the other, two pores corresponding to the removal of 14-atoms were made ("open-end"). The central carbon nanotube is partially doubled by three short carbon nanotubes with the indices $(19,19)$ and $0.5 \mathrm{~nm}$ in length. The radius difference corresponds to the $0.334 \mathrm{~nm}$ distance met in graphite sheets, situated closely, at $0.3358 \mathrm{~nm}$. Two of the short carbon nanotubes are placed at the central nanotube's ends and one is placed $2 \mathrm{~nm}$ away from its open end, which is then inserted in an additional $10 \mathrm{~nm} \times 10 \mathrm{~nm}$ graphene layer, dividing the volume outside the central carbon nanotube into the "filtrate area" and "no gas area". The nanotubes and graphene sheets were generated using VMD [25]. To make the design more applicable to experimental settings, the "no gas area" represents a $2 \mathrm{~nm}$-long pore-free segment for moving the central carbon nanotube through nano gear-based mechanisms. Such may be a very complex undertaking for molecular dynamics studies due to the need of obtaining a constant rotating motion and constant velocity and thus remains to be investigated in future studies. Along the center of the carbon nanotube, four columns of six smaller pores, corresponding to the removal of six atoms making up a hexagonal ring, are present. A gas mixture of $100 \times \mathrm{H}_{2}, 20 \times \mathrm{CH}_{4}, 10 \times \mathrm{CO}_{2}, 10 \times \mathrm{CO}$, $10 \times \mathrm{N}_{2}$, and $10 \times \mathrm{H}_{2} \mathrm{O}$ molecules are placed randomly inside the central carbon nanotube, which is free to rotate around its own axis. 


\subsection{Simulation Details and Force Field Parameters}

All simulations were run using the OPLS-AA force field, together with GROMACS 2018.3. The rotational motion of the central carbon nanotube was achieved using the Enforced Rotation module and iso-pf potential. Three different angular velocities were imposed, with $\omega=180^{\circ} \mathrm{ps}^{-1}$, $270^{\circ} \mathrm{ps}^{-1}$, and $360^{\circ} \mathrm{ps}^{-1}$. For each case, three repetitions were run using the rotational force constant $500 \mathrm{~kJ} \mathrm{~mol}^{-1} \mathrm{~nm}^{-2}$. All simulations were run for 20,000,000 steps, using a step size of $0.5 \mathrm{fs}$ and summing up to $10 \mathrm{~ns}$ simulated time. Coordinates for visualizations were saved every $5 \mathrm{fs}$. Simulation box size was $10 \mathrm{~nm} \times 10 \mathrm{~nm} \times 20 \mathrm{~nm}$ and contained 15,000 atoms. Periodic boundary conditions were active in the $X$ and $Y$ directions. On the $Z$-axis, the top and bottom of the simulation box contained two walls made out of $\mathrm{Kr}$ atoms (Lenard Jones (LJ) 10-4) with a number density of $5 \mathrm{~nm}^{-2}$. In order to confer a repulsive character, interactions with the $\mathrm{Kr}$ atoms were cut at $0.1 \mathrm{~nm}$. VdW and Coulomb cutoff was set at $1.0 \mathrm{~nm}$. The water model used was SPC/E. The Verlet cut-off scheme was used, together with the V-rescale thermostat at $300 \mathrm{~K}$.

Most parameters for the gas molecules were taken from previous studies using the OPLS-AA forcefield [12]. $\mathrm{H}_{2}$ and $\mathrm{N}_{2}$ molecules were modeled as three-site models, with one virtual mass-less atom in the center of the molecules, while $\mathrm{CO}_{2}$ molecules were built as a five-site model, thus using two virtual atoms, as shown in Lemkul's tutorial [26]. $\mathrm{CH}_{4}$ and $\mathrm{CO}$ molecules were built without the aid of virtual atoms and thus contained five, respectively two atoms.

Carbon atoms making up the setup of the membrane were considered uncharged LJ spheres with cross-section $0.34 \mathrm{~nm}$, potential wall depth $0.36 \mathrm{~kJ} \mathrm{~mol}^{-1}, \mathrm{C}-\mathrm{C}$ bond length $1.42 \AA$, C-C-C bending angle $120^{\circ}$, and C-C-C-C planar angles maintained by harmonic potentials with springs constants of $322.55 \mathrm{kcal} \mathrm{mol}^{-1} \AA^{-2}, 53.35 \mathrm{kcal} \mathrm{mol}^{-1} \mathrm{rad}^{-2}$ and $3.15 \mathrm{kcal} \mathrm{mol}^{-1}$ [27].

For data analysis we made use of Python libraries such as NumPy, bokeh [28] and MDAnalysis [29,30].

\section{Conclusions}

We have presented a rotational partial double-walled carbon nanotube hydrogen separation membrane design relying on the recently observed rotational motion of double-walled carbon nanotubes when exposed to an electric field [21] and investigated its gas separation performance under three different imposed angular velocities $\left(180^{\circ} \mathrm{ps}^{-1}, 270^{\circ} \mathrm{ps}^{-1}\right.$, and $360^{\circ} \mathrm{ps}^{-1}$ ), while observing the movement of the gases inside the membrane. We have shown that the outcomes of the simulations are dependent on the structural changes occurring in the rotating membrane and affecting the size and shape of the smaller pores leading to the filtrate area. We have presented a case of very high selectivity for the current setup $\left(\omega=180^{\circ} \mathrm{ps}^{-1}\right)$ and highlighted the advantages of the design such as the fast exit of the $\mathrm{H}_{2}$ molecules from within the nanotube. We have provided explanations for its interesting strong points such as the exit of all $\mathrm{H}_{2}$ molecules within simulated time irrespective of the behavior of the other gases, a phenomenon consistent through all our simulations. Given the significantly different simulation outcomes, we deem that in an experimental setting, tightly controlling the angular velocity would be paramount. Given the imposed angular velocity cases presented, we have shown that the outcome of MD simulations can be both intuitive and counter-intuitive: compared to the $180^{\circ} \mathrm{ps}^{-1}$ case, an increase in the flux of $\mathrm{H}_{2}$ molecules in the $270^{\circ} \mathrm{ps}^{-1}$ case came with a plummeting of the selectivity and allowed many other gas molecules to pass into the filtrate area; however, a further increase of the imposed angular velocity to $360^{\circ} \mathrm{ps}^{-1}$ lead to the exit through the smaller pores of only $\mathrm{H}_{2}$ and $\mathrm{CH}_{4}$ molecules in spite of the increased pore size, making for a very interesting insight useful in gradual separation designs. Compared to other designs, in the presented prototype, the gas mixture was exposed to a large surface area with each gas molecule always traveling towards a pore. In addition, no gas molecules were able to cross back into the membrane.

We have provided flux and permeance estimates and found them comparable to our previous designs and superior to existing experimental solutions [18]. On top of that, our $80 \%$ flux values were found comparable to 12-atoms, 14-atoms, and 16-atoms graphene pores [16]. Thus, we have shown that the angular velocity plays a significant role in hydrogen separation through a rotating nanotube. 
We deem further experimentation involving MD investigations on novel gas separation membrane designs will lead to valuable insights for the hydrogen fuel industry.

Supplementary Materials: The following are available online at http://www.mdpi.com/1422-0067/21/24/9588/s1.

Author Contributions: Conceptualization, M.I. and S.M.; methodology, S.M.; software, S.M.; validation, S.M.; formal analysis, S.M; investigation, M.I and S.M.; resources, M.I and S.M.; data curation, S.M.; writing—original draft preparation, S.M.; writing-review and editing, S.M. and M.I.; visualization, S.M.; supervision, M.I.; project administration, M.I.; funding acquisition, M.I. All authors have read and agreed to the published version of the manuscript.

Funding: This work was supported by a grant of the Executive Agency for Higher Education, Research, Development and innovation funding (UEFISCDI), project number PN-III-P1-1.1-TE-2016-24-2, contract TE 122/2018. Sebastian Muraru acknowledges funding support from a grant of the Ministry of Research and Innovation, Operational Program Competitiveness Axis 1-Section E, Program co-financed from European Regional Development Fund under the project number 154/25.11.2016, P_37_221/2015, “A novel graphene biosensor testing osteogenic potency; capturing best stem cell performance for regenerative medicine" (GRABTOP).

Conflicts of Interest: The authors declare no conflict of interest.

\section{Abbreviations}

MD Molecular Dynamics

\section{References}

1. Bernardo, G.; Araújo, T.; Lopes, T.D.S.; Sousa, J.; Mendes, A. Recent advances in membrane technologies for hydrogen purification. Int. J. Hydrogen Energy 2020, 45, 7313-7338. [CrossRef]

2. Zhu, L.; Xuea, Q.; Li, X.; Jin, Y.; Zheng, H.; Wu, T.; Guo, Q. Theoretical Prediction of Hydrogen Separation Performance of Two-Dimensional Carbon Network of Fused Pentagon. ACS Appl. Mater. Interfaces 2015, 7, 28502-28507. [CrossRef]

3. Bernardo, P.; Clarizia, G. 30 years of membrane technology for gas separation. Chem. Eng. Trans. 2013, 32, 1999-2004.

4. Ockwig, N.W.; Nenoff, T.M. Membranes for Hydrogen Separation. Chem. Rev. 2007, 107, 4078-4110. [CrossRef] [PubMed]

5. Saraswathi, M.S.S.A.; Nagendran, A.; Rana, D. Tailored polymer nanocomposite membranes based on carbon, metal oxide and silicon nanomaterials: A review. J. Mater. Chem. A 2019, 7, 8723-8745. [CrossRef]

6. Li, P.; Wang, Z.; Qiao, Z.; Liu, Y.; Cao, X.; Li, W.; Wang, J.; Wang, S. Recent developments in membranes for efficient hydrogen purification. J. Membr. Sci. 2015, 495, 130-168. [CrossRef]

7. Liang, C.Z.; Chung, T.-S.; Lai, J.-Y. A review of polymeric composite membranes for gas separation and energy production. Prog. Polym. Sci. 2019, 97, 101141. [CrossRef]

8. Tiwari, S.K.; Kumar, V.; Huczko, A.; Oraon, R.; De Adhikari, A.; Nayak, G.C. Magical Allotropes of Carbon: Prospects and Applications. Crit. Rev. Solid State Mater. Sci. 2016, 41, 257-317. [CrossRef]

9. Hirsch, A. The era of carbon allotropes. Nat. Mater. 2010, 9, 868-871. [CrossRef]

10. Monthioux, M.; Kuznetsov, V.L. Who should be given the credit for the discovery of carbon nanotubes? Carbon 2006, 44, 1621-1623. [CrossRef]

11. Novoselov, K.S.; Geim, A.K.; Morozov, S.V.; Jiang, D.; Zhang, Y.; Dubonos, S.V.; Grigorieva, I.V.; Firsov, A.A. Electric Field Effect in Atomically Thin Carbon Films. Science 2004, 306, 666-669. [CrossRef] [PubMed]

12. Jiao, S.; Xu, Z. Selective Gas Diffusion in Graphene Oxides Membranes: A Molecular Dynamics Simulations Study. ACS Appl. Mater. Interfaces 2015, 7, 9052-9059. [CrossRef] [PubMed]

13. Ye, H.; Li, D.; Ye, X.; Zheng, Y.; Zhang, Z.; Zhang, H.; Chen, Z. An adjustable permeation membrane up to the separation for multicomponent gas mixture. Sci. Rep. 2019, 9, 7380. [CrossRef]

14. Sun, C.; Bai, B. Fast mass transport across two-dimensional graphene nanopores: Nonlinear pressure-dependent gas permeation flux. Chem. Eng. Sci. 2017, 165, 186-191. [CrossRef]

15. Shan, M.; Xuea, Q.; Jing, N.; Ling, C.; Zhang, T.; Yan, Z.; Zheng, J. Influence of chemical functionalization on the $\mathrm{CO}_{2} / \mathrm{N}_{2}$ separation performance of porous graphene membranes. Nanoscale 2012, 4, 5477-5482. [CrossRef] [PubMed] 
16. Sun, C.; Boutilier, M.S.H.; Au, H.; Poesio, P.; Bai, B.; Karnik, R.; Hadjiconstantinou, N.G. Mechanisms of Molecular Permeation through Nanoporous Graphene Membranes. Langmuir 2014, 30, 675-682. [CrossRef]

17. Wesołowski, R.P.; Terzyk, A.P. Pillared graphene as a gas separation membrane. Phys. Chem. Chem. Phys. 2011, 13, 17027-17029. [CrossRef]

18. Muraru, S.; Ionita, M. Super Carbonaceous Graphene-based structure as a gas separation membrane: A Non-Equilibrium Molecular Dynamics Investigation. Compos. Part B Eng. 2020, 196, 108140. [CrossRef]

19. Boretti, A.; Al-Zubaidy, S.; Vaclavikova, M.; Al-Abri, M.; Castelletto, S.; Mikhalovsky, S. Outlook for graphene-based desalination membranes. NPJ Clean Water 2018, 1, 5. [CrossRef]

20. Roy, K.; Mukherjee, A.; Raju, M.N.; Chakraborty, S.; Shen, B.; Li, M.; Du, D.; Peng, Y.; Lu, F.; Cruzatty, L.C.G. Outlook on the bottleneck of carbon nanotube in desalination and membrane-based water treatmentA review. J. Environ. Chem. Eng. 2020, 8, 103572. [CrossRef]

21. Bailey, S.W.D.; Amanatidis, I.; Lambert, C.J. Carbon Nanotube Electron Windmills: A Novel Design for Nanomotors. Phys. Rev. Lett. 2008, 100, 256802. [CrossRef]

22. Feng, J.-W.; Ding, H.-M.; Ren, C.-L.; Ma, Y.-Q. Pumping of water by rotating chiral carbon nanotube. Nanoscale 2014, 6, 13606-13612. [CrossRef] [PubMed]

23. Tu, Q.; Yang, Q.; Wang, H.; Li, S. Rotating carbon nanotube membrane filter for water desalination. Sci. Rep. 2016, 6, 26183. [CrossRef] [PubMed]

24. Rahman, M.; Chowdhury, M.M.; Alam, K. Rotating-Electric-Field-Induced Carbon-Nanotube-Based Nanomotor in Water: A Molecular Dynamics Study. Small 2017, 13, 1603978. [CrossRef] [PubMed]

25. Humphrey, W.; Dalke, A.; Schulten, K. VMD: Visual molecular dynamics. J. Mol. Graph. 1996, 14, 33-38. [CrossRef]

26. Lemkul, J.A. From Proteins to Perturbed Hamiltonians: A Suite of Tutorials for the GROMACS-2018 Molecular Simulation Package [Article v1.0]. Living J. Comput. Mol. Sci. 2019, 1, 5068. [CrossRef]

27. Chen, J.; Wang, X.; Dai, C.; Chen, S.; Tu, Y. Adsorption of GA module onto graphene and graphene oxide: A molecular dynamics simulation study. Phys. E Low Dimens. Syst. Nanostruct. 2014, 62, 59-63. [CrossRef]

28. Bokeh Development Team. Bokeh: Python Library for Interactive Visualization. 2020. Available online: https://bokeh.org (accessed on 10 October 2020).

29. Gowers, R.J.; Linke, M.; Barnoud, J.; Reddy, T.J.E.; Melo, M.N.; Seyler, S.L.; Domanski, J.; Dotson, D.L.; Buchoux, S.; Kenney, I.M.; et al. MDAnalysis: A Python Package for the Rapid Analysis of Molecular Dynamics Simulations. In Proceedings of the 15th Python in Science Conference (SciPy), Austin, TX, USA, 11-17 July 2016; pp. 98-105. [CrossRef]

30. Michaud-Agrawal, N.; Denning, E.J.; Woolf, T.B.; Beckstein, O. MDAnalysis: A toolkit for the analysis of molecular dynamics simulations. J. Comput. Chem. 2011, 32, 2319-2327. [CrossRef]

Publisher's Note: MDPI stays neutral with regard to jurisdictional claims in published maps and institutional affiliations.

(C) 2020 by the authors. Licensee MDPI, Basel, Switzerland. This article is an open access article distributed under the terms and conditions of the Creative Commons Attribution (CC BY) license (http://creativecommons.org/licenses/by/4.0/). 